\title{
Emergency Medicine in China
}

\author{
Xiang-Yu Hou \\ School of Public Health, Queensland University of Technology, Brisbane, \\ Department of Emergency Medicine, Royal Brisbane and Women's Hospital, Brisbane, \\ School of Medicine, The University of Queensland, Brisbane, \\ Australia
}

\section{Introduction}

With the world's largest population of 1.3 billion, China is a rapidly developing country. In line with this development, China's enormous health system is experiencing an unprecedented series of reforms. According to a recent official government report, China has 300, 000 health organizations, which include 60, 000 hospitals and a total number of 3.07 million beds (China NBoSoP 2006). To provide health services for the national population, as well as the substantial number of visitors, China has 1.93 million doctors and 1.34 million registered nurses (China NBoSoP 2006). From 1984 to 2004, the number of inpatients grew from about 25 to 50 million, with outpatient figures increasing from 1.1 to 1.3 billion (China $\mathrm{MoH}$ 2006). The scale of the health system is likely bigger than in any other countries in the world, but the quality of medical services is still among the levels of developing countries. In 2005, approximately $3.8 \%$ of inpatients (about 1.5 million)(China NBoSoP 2006) were admitted because of injury and poisoning, which created significant load for the acute health system. These increased figures are at least partly because of the development of the health system and technological health-care advances but, even with such advances, this rapid change in emergency health-care demand has created a very significant burden on existing systems.

The Chinese Emergency Medical Service system is primarily composed of three sectors: prehospital care, hospital Emergency Departments (EDs) and hospital intensive care units (ICU). While pre-hospital care systems development in China, especially the workforce status, has been reported and discussed before (Hou and Lu 2005), the purpose of this book chapter is to introduce the development of emergency medicine (EM) in China, including the role of traditional Chinese medicine, which is based mainly on hospital ED. EDs in hospitals are like the window of the world where the health problems in the community would show among the ED patients (Hou and Chu 2010), therefore an in-depth understanding of the hospital EDs in different countries would enhance the appreciation of the general health system in other countries.

\section{Brief history of emergency medicine development in China}

Development of Chinese hospital ED over the past two decades has been characterized by two main factors: establishment of a professional body of Emergency Medicine (EM) 
practitioners and the institution of ED in all county-level hospitals nationally. The professional association of EM was officially founded in 1986, as a recognized specialty branch of the China Medical Association. This EM branch has eight specialty groups, including prehospital, resuscitation, trauma, intensive care, acute poisoning, paediatric EM, disaster medicine and ED quality control (Jiang 2004). Professor Jiang has described EM as consisting of three integral and interrelated sections - prehospital care, hospital ED and critical care in ICU wards (Jiang 2004). From the late 1990s onward, all county-level hospitals in China established ED, with urban hospitals having already done so through the 1980s. For example, Tongji Hospital in Wuhan city (a relatively well-known large tertiary hospital) established its ED in 1986 (Bai, Li et al. 2004). According to its Health Bureau, even Guangxi Province, a relatively low socioeconomic area, had established a Department of Emergency Medicine in all county-level hospitals by 2003 (Lu and Qin 2005).

At pace with the rapid economic development of the country, Chinese hospital EDs have been equipped with 'hardware' equipment that is often state-of-the-art. However, because of underdeveloped 'software', such as clinical and technical expertise and fully functional management systems, equipment might not be used or maintained appropriately. This disparity has occurred largely because of an outdated economic health-care system that has not kept pace with the political and socioeconomic change in China.

A unique characteristic of the health care system in China is that Traditional Chinese Medicine (TCM) and western medicine are practised at almost all levels of the health care delivery system including hospital EDs. Almost every city in China has a TCM hospital, and almost every TCM hospital has an ED. Among the hospitals practising western medicine approximately $95 \%$ have a TCM outpatient clinic as well as an inpatient ward (Hesketh and Zhu 1997). There were over 300,000 TCM practitioners in China in 1995 and the number could be grown significantly since the Chinese central government continued its policy to expand TCM in China (Hesketh and Zhu 1997). The rapid expand of hospital EDs inevitably caused some concern in risk management at some county level TCM hospitals (Liu 2010) which could be due to the problems such as young nurses working at the front line who did not have enough working experiences in dealing with acute illness (Liu 2010), underresourced in the number of clinicians and equipment, no standardised clinical procedure and not sufficient herbal remedies for their hospitals (Wu 2009).

It is not only the political will, but also the grass root demand among the general population of Chinese, that made the further grow of TCM possible. It was reported that in 1992 in Jiangsu province ( a relatively richer and more sophisticated province in China) about 10 million patients chose to be treated by TCM (not western medicine) at hospitals (Hesketh and Zhu 1997). It was reported that TCM treatments including herbal remedies, acupuncture, massage, and moxibustion, accounted for approximately $40 \%$ of health care delivered in China (Hesketh and Zhu 1997). For example, the First Affiliated Hospital ED at Guangzhou TCM University, established in 1965 (Zhou 1996), has a historical record showing the process how TCM saved a patient's life from snake bite. This is an example of evidence regarding the perception of TCM among the general population in China.

It needs to be noted that TCM has always been part of the Chinese history and culture. Here is an example to illustrate this. The author of this chapter's first name is Xiang-Yu (Pinyin from the Shandong dialect, and Xiang-Ru in mandarin) which is a Chinese herb 香薷 (picture below) that all its leaves, flowers, and roots can be used for herbal medicine treating 
patients with a range of health problems. The author's father, who was a school teaching in Chinese literature, hoped that his daughter would make the society a better place, just like this herb to the patients.

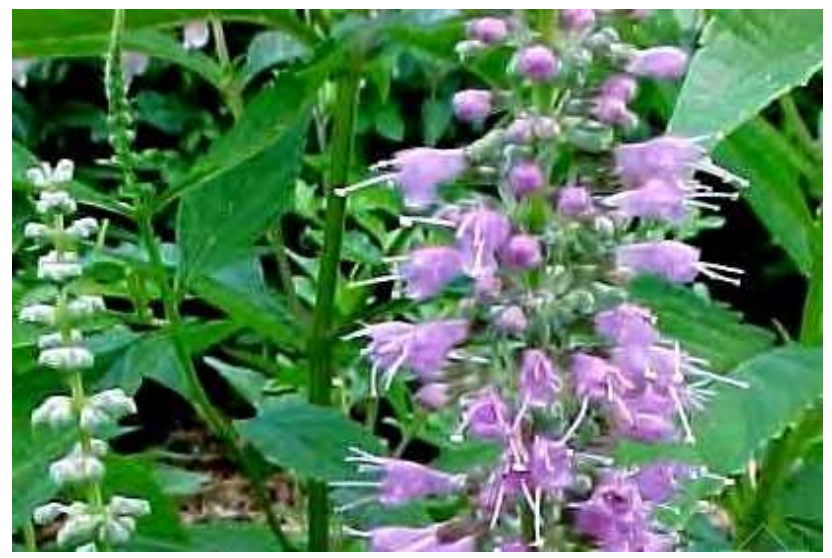

The Chinese herb Xiang-Ru

Therefore, TCM has been a well accepted practice among the Chinese population for over thousands of years. Under this macro environment combined with political will, there is no doubt that a profession will develop speedily when political will and population demand are available. Dept of TCM at China Ministry of Health held a forum in Chongqing city in November 1983 to discuss the development of Traditional Chinese Emergency Medicine (TCEM) in all TCM hospitals in China (Luo 2010). There were seven groups established during that Forum to focus on different acute health problem. This was later formally announced in 1985 at a Shanghai TCEM Meeting (Luo 2010). The focus groups included high fever, stroke, stomach-aches, and blood group. Among each group, there were $8-10$ provincial TCM hospitals to lead research related activities (Luo 2010). Two years later, the first China national scientific TCEM conference was held in Changchun in 1987; followed by the second conference in 1992 in Guangzhou (Luo 2010). Through a lot of hard work and discussion among the TCEM professionals, the TCEM practice guideline was then finalised and published in 1996 (Luo 2010).

A couple of years later, the Emergency Medicine Branch of China Traditional Chinese Medicine Association was established in 1997 (Chao, Jiang et al. 2010) with eleven TCEM Centres assembled in all over the country. This was officially announced on the $5^{\text {th }}$ January 1998 (Luo 2010).

Here is an example to demonstrate the rapid development of TCEM in China. The Jiangsu Province TCM Hospital ED was established on the $5^{\text {th }}$ June 1963, with a couple of TCM practitioners (Xi 2008). By 2006, the number of ED patients was over 100,000. It was reported that the successful rescue rate was approximately 92\% (assuming general mortality at this ED in 2006 was about $8 \%$ ), and the TCM treatment was applied to $90 \%$ of the patients (Xi 2008). By 2008 this TCM hospital ED had 60 clinic beds, 12 beds in observation ward, and four ICU beds. Considering the relatively small market for TCM in the health system in modern China, this Jiangsu Province TCM Hospital ED is an example of the rapid development of TCEM in China. 


\section{Current emergency medicine practice among Chinese hospitals}

The current ED practice among hospitals in China will be discussed in three parts: financial, clinical and education and training of health professionals. This is mainly due to the availability of published literature and its importance in affecting the ED practice in hospitals in China.

\section{Part I: the financial situation of China hospital ED}

As a result of comprehensive macro-level trade and economic reform since the 1980s, China has essentially developed a market economy. The effect of this economic reform on the health-care system was that the Chinese government dramatically reduced hospital funding, from almost $100 \%$ of the premium to about $10-20 \%$. The $80-90 \%$ balance of hospital premiums are now borne by the patients themselves, who are obliged to pay for most of their own health-care costs through a fee-for-service system (Yang, Huo et al. 1999). Figure 1 was a picture taken at a teaching hospital ED in Beijing which is a notice to all ED patients saying they need to purchase a card to process payment while at ED and the deposit for the card could be refunded when leaving ED. Thus, the number of patients attending any hospital and the value of services rendered directly determine the hospital's income and viability. With about $40 \%$ of a typical hospital's income resulting from emergency presentations, responsibility for ensuring viability of hospital services rests largely with ED (Yang, Huo et al. 1999).

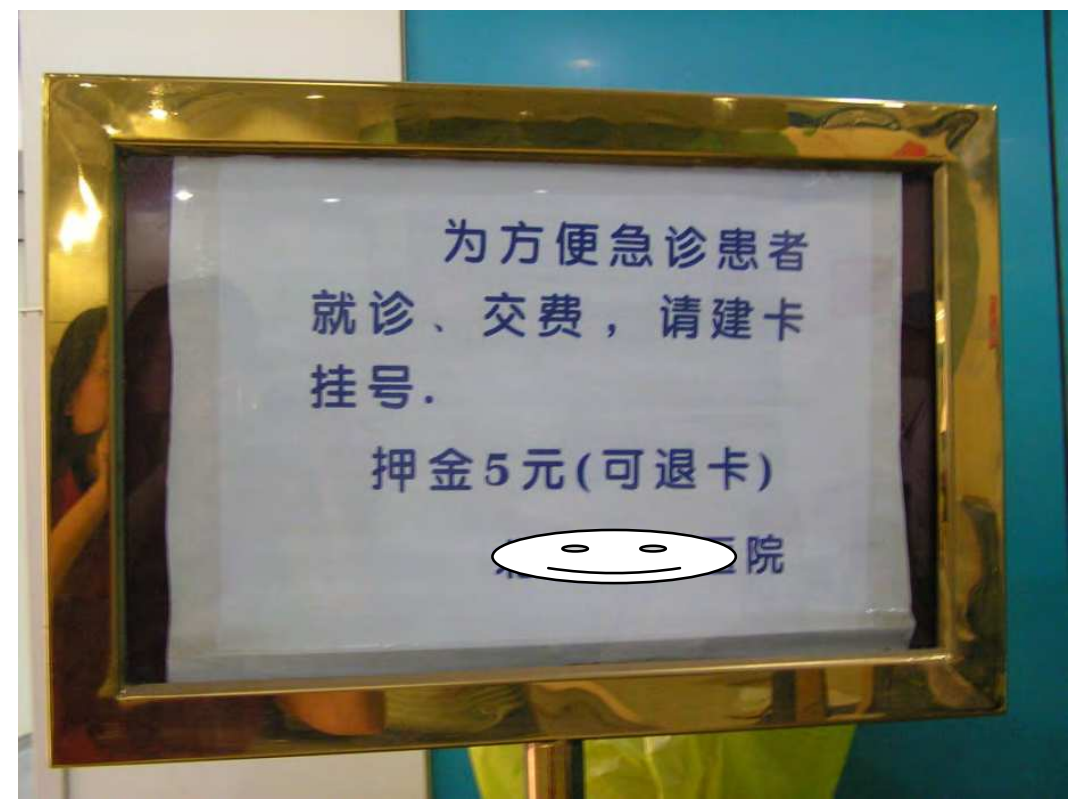

Fig. 1. Notice to ED patients about establishing a card for payment.

Further, financial 'success' of hospitals has become fundamentally linked to effective marketing strategies, particularly those highlighting emergency services. The need for hospitals to survive in a competitive health-care market has significantly contributed to the 
establishment and effective marketing of ED in Chinese hospitals. For example, Shanghai Changhai Hospital, Shanghai, China, has been enormously successful in the fee-for-service health-care market, and has attributed this success to factors such as quality of ED clinical services, patient-focused service, reasonable costs, media and marketing (Yang, Huo et al. 1999). In addition, its success is thought to be essentially related to provision of aesthetic features in the ED, including large-screen televisions in the intravenous therapy day-room, a facility that services around 500 patients per day (Yang, Huo et al. 1999). Such factors are believed to have accounted for around a 10\% annual increase in this hospital's ED patient number (Yang, Huo et al. 1999). Figure 2 provides a general idea what an intravenous therapy day-room is like in China hospitals. At the other end of the spectrum, negative publicity resulting from emergency clinical mistakes can be devastating for hospital business, and is actively avoided.

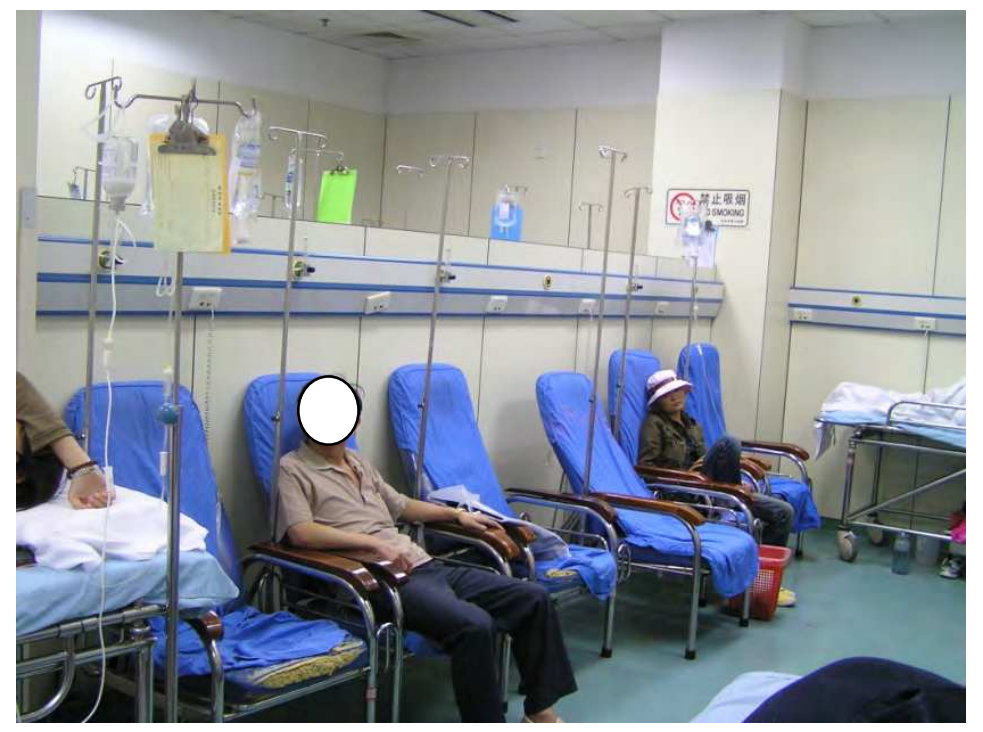

Fig. 2. Intravenous therapy day-room.

Therefore, to make patients happy becomes the first priority for the medical service providers. This is simply because those happy patients will come back to the same hospital to pay for the medical services again, then reputation spreads and more financial return is feasible.

When we visited a few EDs in Shenyang city in China in 2006, we did question the ethical issues of providing intravenous antibiotics to common cold or influenza patients. We were told by the doctors that they are well aware of the potential problems in overusing antibiotics, but the demand from the patients makes it impossible to refuse as 'Clients are God' in a market system plus that the direct financial return for their hospitals is also a consideration. The bigger size \& better service from the hospital ED, the more crowded the hospital ED partly due to the market mechanism. Figure 3 and 4 were taken from a tertiary teaching hospital ED in Beijing where patients were treated in the corridor or intravenous treatment room where usually no beds are available. 
In summary, the market system and financial responsibility of ED to their hospitals make it difficult for emergency health-care providers to develop and deliver a high-quality clinical service to their patients.

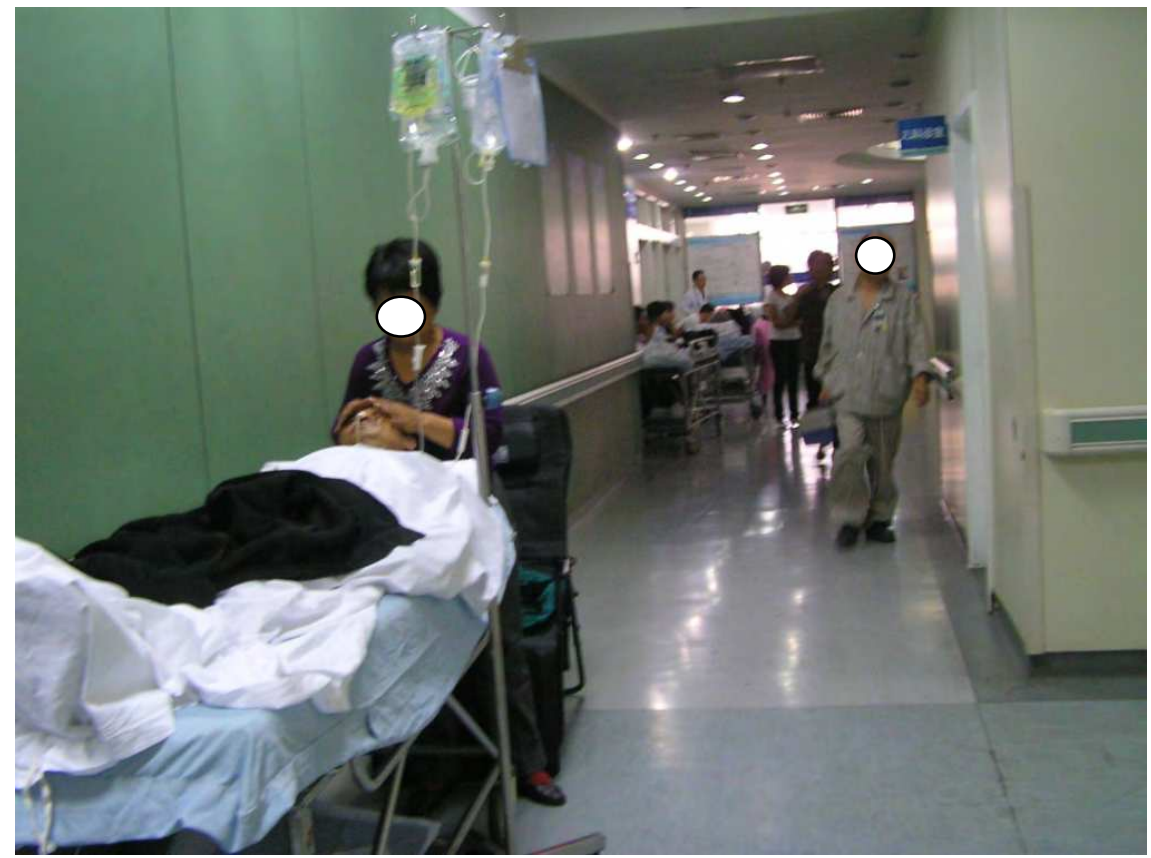

Fig. 3. ED patient being treated at the corridor.

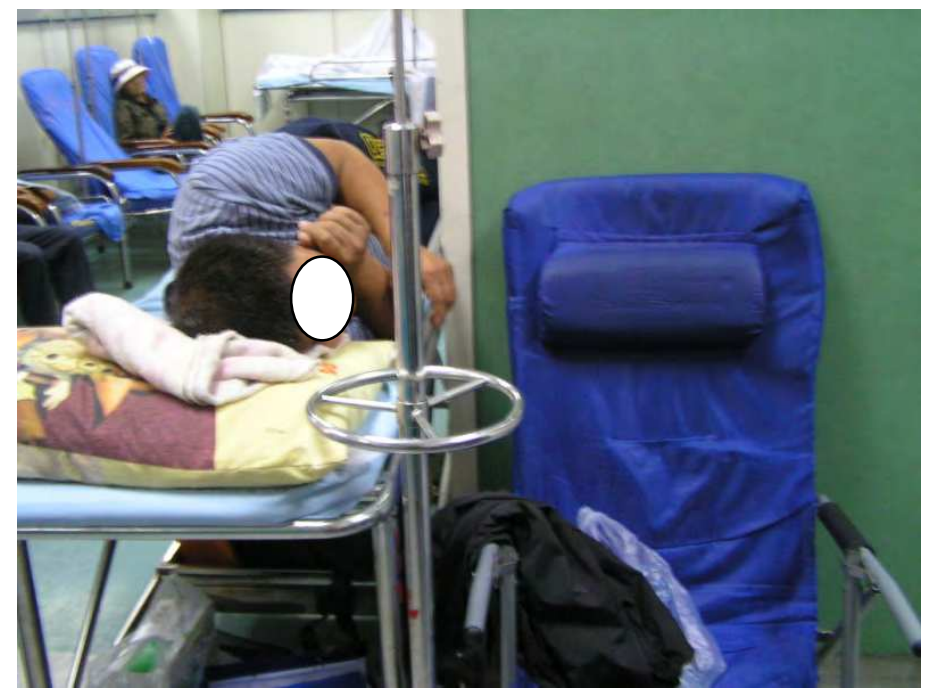

Fig. 4. ED patient on a temporary bed at an IV room. 


\section{Part II: the clinical practice situation of China hospital ED}

The current configuration of hospital ED in China reflects the conceptualization of these units as places from which patients can be allocated to appropriate departments as soon as possible, with little treatment actually provided in the ED themselves. The concept of ED as a kind of 'transit lounge' has resulted in the coining of the term 'Green Channel' by Chinese health professionals (Luo 2003). The Green Channel implies rapid transit to the inpatient services for patients, with little intervention in the ED, like green lights in the traffic. Dr Luo, from Zhejiang University, one of China's leading education institutions, has described emergency clinicians' dissatisfaction with the 'Green Channel' ED conceptualization (Luo 2003). Other surveys (e.g. two studies (Wang 2000; Liu, Xie et al. 2002) have also highlighted clinicians' concerns that patients awaiting allocation to other departments often suffer complications, arising from delays and disagreements between departments about which will receive them. This was believed to be the case even for trauma patients and patients with life-threatening conditions, resulting in considerable preventable mortality (Wang 2000). Figure 5 is an example of a hospital ED where different health problems are allocated to different departments. For example, Department One was for neurological medical problems, Department Seven was for neurological surgical problems, and Department eight was for orthopaedics problems.

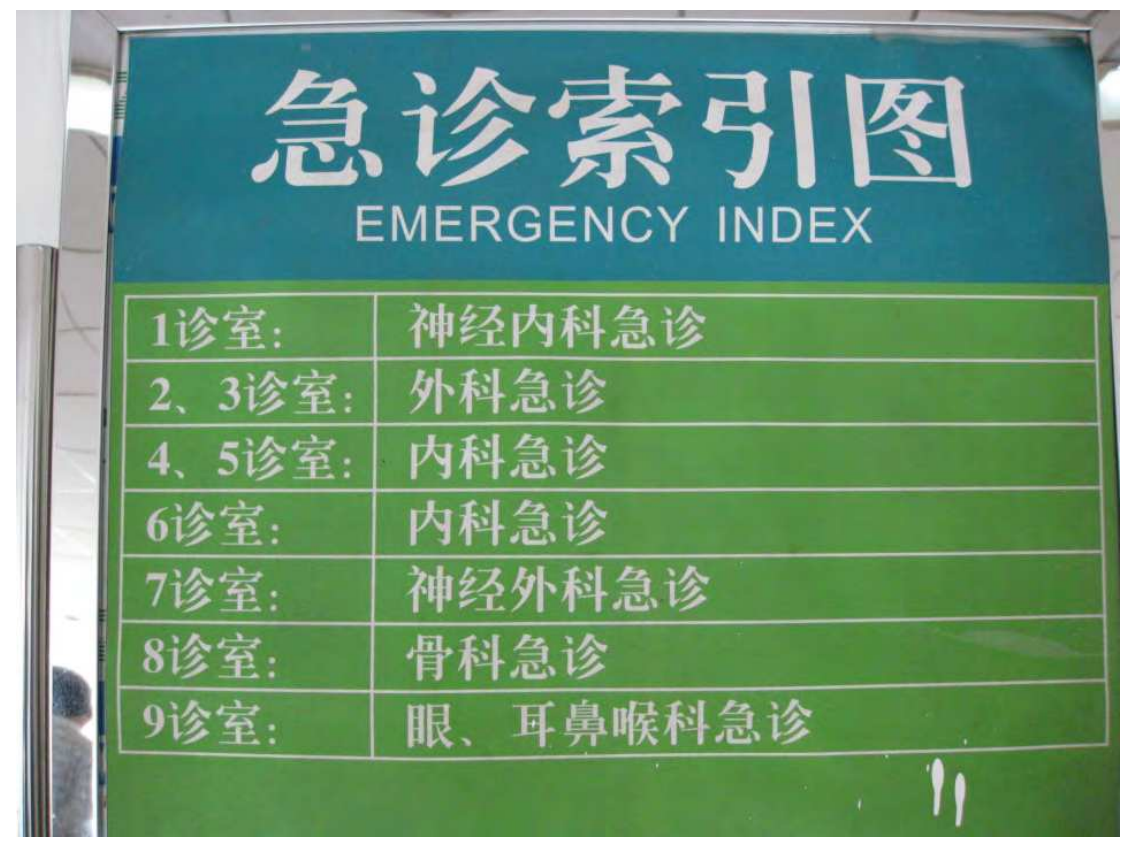

Fig. 5. An ED index directory informing patients which room to go for what type of emergency problem.

Another issue is the limited facilities available in many EDs, largely because of the 'Green Channel' concept described above. For example, only 38\% of EDs in county-level hospitals in Guangxi Province had surgical facilities in 2005 (Lu and Qin 2005). This is largely because 
patients requiring such treatment would be expected to be transferred to surgical departments immediately following their arrival in the ED, rather than being assessed in the ED and prepared for the operating theatre there. For example, a survey of a convenience sample of 2183 patients presenting to the Shanghai Ruijin Hospital ED (a leading hospital ED in Shanghai) in June 2008 showed that following triage, patients were predominantly referred to an internist (41\%), neurologist $(14 \%)$, pulmonologist $(11 \%)$, or general surgeon (9\%) (Lammers, Folmer et al. 2011). One of the founders of Chinese EM, Professor Yitang Wang, has argued that future ED should include full surgical facilities, including operating theatres (Wang 2001). This sentiment has been supported by other scholars since (Sun, Tang et al. 2006).

The body of knowledge in EM in China is somehow behind the world as well. For example, a research paper written by a leading expert in EM in China and published by the national leading EM peer-reviewed journal, discussed the concept of triage. The article showed a lack of understanding of the importance and role of triage in emergency and disaster management (Wang 2002).The author believed that just to rush all the victims to a hospital would be the right strategy if there were many severely damaged patients and a hospital close to the site. This level of triage awareness demonstrated the underdeveloped body of knowledge in EM, as the statement from a developed country in 2002 described that the most significant advance in EM has been formalization and application of triage (Jelinek, Cameron et al. 2002).

Another example of the low standard in clinical practice in China is in the area of chemical poisoning treatment. Chemical poisoning is a leading cause of poisoning patients attending the ED in hospitals in China. Averagely about 500 patients attend each hospital in China (Zhou, Chen et al. 2004). Most alcohol poisoning happened in the east, west and south parts of China whereas most carbon oxide poisoning happened in middle and north part of China (Zhou, Chen et al. 2004). There are an estimated 170, 000 deaths annually from pesticide poisoning, mainly from Organic Phosphates (OP)(Little and Murray 2004).The mortality of OP pesticide poisoning in China was about $10 \%$ whereas overseas report of $2 \%$ (Wang, Wang et al. 2006). The author reported that among the $10 \%$ of patients who died, $60 \%$ of them died of atropine poisoning when atropine was overdosed during treatment. The consensus guidelines on reasonable precautions that should be taken when managing these patients, developed by Dr Little, could be of significant help to Chinese doctors (Little and Murray 2004).

The current clinical practice standards in China hospital ED are alarming when considering a pandemic situation. To apply World Health Organization's international standards, such as the International Health Regulations 2005 (Bradt and Drummond 2006), China needs to develop its EM practice to the world's standard as fast as possible. Developed countries with mature EM practice, such as Australia, New Zealand, USA and UK, could contribute to this development significantly to benefit the global community of emergency medicine professionals.

\section{Part III: the education and training situation of China hospital ED}

Although there have been dramatic improvements and advancements in recent years, the Chinese education and training system for Emergency Medicine is not yet fully developed. Thus, insufficient numbers of high-quality EM specialists have thus far been produced. 
Another factor that limits the development of the specialty is the fact that doctors working in the ED often do not do so as their primary area of specialization. They are commonly specialists in other departments, such as medicine and surgery, who work in the ED on a rotating roster. The proportion of doctors and nurses who are employed to work in the ED on ongoing appointments varies across hospitals, and has been estimated to be from $50 \%$ (Xu 2000) to 60\% (Luo 2003). This situation causes problems for the career development of clinicians and the absence of a clear career path for those aspiring to specialize in Emergency Medicine is likely to act as a deterrent. A recent survey found that Chinese clinicians clearly felt that this was the case (Bai, Li et al. 2004).

Three different types of Chinese hospital EDs have been described: dependent, semidependent and independent, with the type determined by allocation of human resources (Fan, Li et al. 2000). In the 'dependent' type of ED, only the ED director holds an ongoing appointment, with all other staff rostered on a rotational basis from other hospital departments. In the 'semi-dependent' type of ED, the director and some other staff members hold ongoing ED appointments. On the other hand, 'independent' types ED are staffed completely by doctors and nurses with ongoing ED appointments. Fan et al. surveyed 53 tertiary-level hospitals in China (termed San-Ji-Jia-Deng in Chinese), with an average inpatient bed number of 895 , average day patient number of 2,168 and mean staffing of 1,556 health professionals. Among all of these hospitals, only three were identified as having 'independent' type ED, highlighting the slow development in terms of the Chinese EM workforce (Fan, Li et al. 2000).

Evidence-based training and practice are a concept that has been welcomed and widely accepted by Chinese clinicians (Song and Fan 2003). Consequently, educational institutions have been involved in a complete overhaul of their curricula over the past 20 years, and many courses at Chinese universities can now be described as among the best in the world. However, specialty training in some postgraduate areas is still often sought abroad, in part as a result of underdeveloped courses and academic expertise.

Tertiary educational programs in Emergency Medicine in China began in 2002 with the establishment of an EM major within the clinical degree program in Nanjing Medical University (Wang 2002). However, it has been acknowledged that one of the main reasons for the continuation of such majors is to ensure a supply of qualified ED staff, as the perception remains that ED is one of the least attractive hospital areas for medical graduates, despite the fact that ED doctors could master the essential clinical skills including eight medical skills and eight surgical skills (Wen 2005).

It was proposed that it takes three years for a clinician with a doctoral degree, and 5 years if bachelor or masters degree, to specialize as a specialist in Emergency Medicine (Bai, Li et al. 2004). However, the duration of training is still an question of debate, as some experts believe that it takes five years to master the necessary knowledge and skills and it makes no difference whether you have a bachelor or master degree (Wan and He 2006). The content of the training program has not been standardized in China, which varies in time duration and content of training in a range of departments in hospitals (Wan and He 2006).

It was argued among the Chinese health professionals whether it was appropriate to develop a major of EM in clinical medicine (Song 1999). The argument result was summarized in Table 1. 


\begin{tabular}{lll}
\hline & $\begin{array}{l}\text { Specialists of emergency } \\
\text { medicine }\end{array}$ & $\begin{array}{l}\text { Specialists in other clinical areas } \\
\text { and roster in ED }\end{array}$ \\
\hline Cost/benefit ratio & Low & High \\
Clinical service quality & General high & General low \\
& Specific case low & Specific case high \\
ED management & Easy & Difficult \\
EM as a specialty to develop & Fast & Slow \\
Acceptance & Difficult & Easy \\
To develop & Initially difficult & Initially easy \\
& Later easy & Later difficult \\
\hline
\end{tabular}

Table 1. With or without emergency medicine specialists in hospital EDs.

The author is from Hainan Province People's Hospital, Hainan, China, and actually supported the idea of developing EM as a specialty area among clinical medicine in China (Song 1999).

China is not a leading country in Asia in developing EM in many ways, but is considerable ahead of some countries, such as India (David 2007) where the medical system is governed by a single, central, regulatory body known as the Medical Council of India (MCI) which oversees all specialities. Multiple representations have attempted to enlighten MCI regarding the need for postgraduate training in EM. However, no positive notes of response have been forthcoming, by November 2006 (David 2007). The author described that without the consent of the $\mathrm{MCI}$, a recognized postgraduate training in EM cannot be started in the country (David 2007), while emergency physicians cannot play a significant role in persuading the government.

By all estimates, the pace of growth of EM in China will continue to accelerate as China's economy and demographics approach those of the west, but the final form they will take is still uncertain (Ali 2001). The certain point is that China is taking the parts of the western system it can use and implementing its own methods in the overall practice of EM (Clem, Thomas et al. 1998).

\section{Traditional Chinese Emergency Medicine (TCEM) in hospital EDs}

\subsection{Brief theory and current situation}

Traditional Chinese Medicine (TCM) is based upon descriptive metaphors for human energies that do not translate well into the English terms, and very often some meanings are lost in the process of translation. The basic concept in TCM is that a vital force of life surges through the body called Qi. Any imbalance in Qi can cause illness or diseases. TCM focus on the whole body, not just the foot or head where the illness is located (Zhang, Wang et al. 2007). For example, pain is the result of conflict between blood and Qi (Dillard and Knapp 2005). The treatment in pain using TCM would then include balancing the energy, nourishing the blood, and building up deficient blood using herbal remedies, acupuncture, massage, and moxibustion.

The importance of TCM application in Emergency Departments at TCM hospital in China has been demonstrated by some academics who believe that "no western medicine if TCM works; and TCM first, western medicine later" (in Chinese, it says “能中不西, 先中后西”) 
(Wu 2011), seeing western medicine as the "opposition". However, some TCM clinicians are more positive towards western medicine and believe that western medicine could compliment TCEM in diagnosis and treating acute illness and trauma patients (Liu 2008) which may be due to the slow development in TCEM regarding its limited facility and equipment and staff's low capacity (Su, Ding et al. 2002). It is not surprising that some TCM practitioners, maybe the most of TCM practitioners, believe that clinicians should use whatever the best for the patients whether it was TCM or western medicine or both (Sun and Xi 2006 ) (Su, Ding et al. 2002).

To apply the essential concept of TCM as the holistic approach in treating a patient, the nurses in charge of PCI patients practised the "emotional nursing" which was to look after the patients prior, during, and post the operation regarding their emotional status and concerns. It was demonstrated that the patients' general outcome was improved after the intervention (Wang 2011). Similar outcome was also reported in PTCA older patients (Liu and Lin 2009), the hysteria patients (Cheng and Lu 2011) and suicidal attempt patients attending the emergency departments in a TCM hospital (Zhou 2007).

Similar to the development in emergency medicine among the emergency physicians, doctors specialising in Traditional Chinese Emergency Medicine (TCEM) should be able to deal with all acute illness and trauma whether it was medicine, surgery or obstetrics problems (Jiang, Chen et al. 2007) which was supported by the ED development at Guangdong Province Fe-Shan TCM hospital (He, Zhang et al. 2004).

Due to the large number of universities delivering western medicine courses, most ED doctors working in TCM hospitals have the degree in western medicine, which equals the Bachelor of Medicine and Bachelor of surgery (MBBS) program in medical schools in Australia. For example, in Kunming City TCM Hospital ED, there were 20 doctors. Fifteen of them were trained in western medicine, and 12 out of these 15 doctors graduated with the second degree in TCM (Liu, Xie et al. 2002).

Herbal medicine is a large part of TCM. China TCM Management Bureau, the highest level of TCM professional organisation in China, publishes the recommended herbal remedies list for Emergency Departments at TCM hospitals. There were 15 products in the list published on 9th January1993 (Wu 1997), and 50 in 1997 (China-TCM-Management-Bureau 1997). The list has been updated every two years. There were about 134 injection type of herbal remedies being used in western medicine practice in China (Luo 2010). These herbal remedies for injection use included anaesthetic, anti-bacteria, anti-shock, treating coronary heart problems, pain reliever, stop-bleeding, anti-cancers and improving immune system (Luo 2010). It was believed that a well performed TCM hospital ED should use all the products in the list regularly, and that Chongqing TCM Hospital ED used about $42 \%$ of the list products (Li, Cheng et al. 2006) was regarded as a low level performance and needed to increase the number of the used herbal remedies rapidly.

\section{Diagnosis and treatment}

The traditional diagnosis method in TCM is called Four Diagnosis Method, namely inspection (望), listening and smelling（闻），inquiring（问） and palpation（切）(Wei and Su 2010). Applying this "Four Diagnosis" method in triage in emergency medicine in TCM hospitals (Chen 2010), its accuracy rate was $90.3 \%$ as good as non-TCM hospitals 
(Zhang 2007). This finding was supported by a report about Four Diagnosis method applied in Triage for abdominal pain patients (Tao 2003).

It was reported that there were three ways to make a diagnosis in TCEM: to borrow the western medicine diagnosis; to use the TCM diagnosis only; and to combine TCM with western medicine to generate a diagnosis (Liu, Wang et al. 2010). It would not make any sense for a western medicine practitioner to read a diagnosis in TCM. For example, diagnosis of unconscious patients in TCM has six types, all of them are in TCM terms and no translation to English was available to understand them (Zhang 2010). The author of this book chapter was educated in western medicine in China and has very little idea what the diagnosis really mean regarding the concept of diagnosis in western medicine. This "confusion" among the practitioners trained in western medicine was also supported in a general discussion paper about principles of diagnosis in TCEM (Zhong 1983). It would not surprise the western medicine colleagues that misdiagnosis would be more often in TCM, which could be a result of the many factors including the limitation in TCEM diagnosis; limitation in herbal remedies treatment; weak concept of "emergency"; and under-equipped with modern facilities (Liu and Cui 2004).

Despite the confusion in understanding the TCM diagnosis, its application in successfully treating a range of acute illness for patients attending TCM hospital EDs has been reported in the Chinese literature. These include dizziness patients (Lan and Lin 2009), headache, and head injury patients (Gao 2009) (YAN, HAN et al. 2008), nose bleeding (YAN, HAN et al. 2008), chest pain patients (Shen and Han 2009) (Gao 2009), acute myocardial infarction patients (Wang and Chen 2009), intoxicated carditis with shock (Tan and Mei 1995), haemorrhoid (Deng and Zheng 1997), acute pneumonia and acute pancreatitis, (YAN, HAN et al. 2008), pain from cancer (Xu and Liu 1994) and even fracture (Jin 1982). It was reported that acupuncture was effective and fast in treating acute stomach pain, high fever, hysteria, and hiccup (Ni 1998); and acupressure was impressively effective in treating angina, hiccup and cough (Jiang, Tang et al. 1992).

In many cases, the treatment for the patients attending a TCM hospital ED was a combination of TCM and western medicine. Researchers believe that the application of TCM in treating SARS (Severe Acute Respiratory Syndrome ) in 2003, swine influenza in 2004 and H1N1 influenza in 2010, had demonstrated the feasibility of combining TCM with western medicine to improve the treatment outcome for patients with virus infections (Luo 2010) (Jiang, Tang et al. 1992).

\section{Teaching}

The general perception is that where there is a university teaching western medicine, there is a university teaching TCM. For example, in the capital city of Beijing, there is a Beijing Medical University (now called Health Science Centre of Peking University) and there is also a Beijing TCM University. Therefore, there are quite a few publications in Chinese language about teaching TCM and its vital role in keeping the TCM practice alive and continues to develop. It needs to be noted that universities in China now have a major in Chinese-Western combined medicine where students learn both the TCM and the western medicine (Sun and Xi 2006 ); and that the first group of doctorate students undertaking research in the area of Traditional Chinese Emergency Medicine at Guangzhou TCM 
University graduated in 1996 (Zhou 1996). These are important milestones for the TCEM teaching and research in China.

Regarding the teaching content in knowledge, it was important to teach the intern students at Emergency Department in TCM hospitals the essential knowledge of the Four Diagnosis in TCM, especially now most county TCM hospitals have emergency departments (Zhou and Zhang 2011) and the recognition of these skills in ED triage (Lin and Liu 2003). It was recommended to combine the concept and theory of TCM with western medicine $(\mathrm{Wu}$ 2011). Some leading experts in TCM believe that an essential part to further develop TCEM was to ensure the TCEM practitioners are experts in western emergency medicine although that how to achieve this objective was still unclear (Jiang 2009). For example, to treat bacteria infectious patients with fever, both antibiotics to kill the bacteria and herbal remedies to build the body's immune system should be considered (Wu 2011). However, in reality of current teaching practice, the education and training of western medicine in TCEM teaching was not sufficient enough to provide students enough acute clinical skills, partly because some TCM clinicians tend to only use TCM in treating acutely ill patients (Zhang, Zhang et al. 2010). Nurses are important part of this team and it is also important to train the nurses for the core capacity at ED in TCM hospitals (Chen, Wang et al. 2011) as well as their capacity in triage and legal practice (Chang, Pang et al. 2010).

Regarding the teaching content in skills, reports suggest that students graduated from TCM universities have a good understanding of the TCM theories but low clinical practice skills at EDs which explained why a standard set of teaching and training criteria was required for the intern students at ED in TCM hospitals (Li 2011). Some academics believe that students' critical thinking capacity is as important as their emergency medicine clinical skills $(\mathrm{Wu}$ 2011), which would be accepted by most western medicine practitioners.

Regarding the teaching method, it was reported that to employ Problem Based Learning method in teaching TCEM could activate students' interests in studying this area, improve their general understanding of the subjects, and enhance their critical and clinical thinking capacity (Li, Yang et al. 2010) (Li, Han et al. 2009).

Continuing professional education is important for any health profession. To send TCEM practitioners in low level TCM hospitals to western medicine hospitals to learn and update their knowledge, skills and practice in western emergency medicine was regarded as an important measure for the TCEM development strategy (Ding 2005).

\section{Research}

Published literatures in Chinese language in the area of Traditional Chinese Emergency Medicine (TCEM) are relatively few and without strong research designs in the concept of western medicine research. Some TCEM researchers in China even believed that it is not appropriate to apply western medicine methods to assess the effectiveness of the TCEM treatments (in Chinese, it says“道不同理能清吗?”) and that the challenges from western medicine was a barrier for the TCEM development in China (Chao, Jiang et al. 2010).

Here is an example to illustrate the research status of TCEM in China. Almost ten out of ten clinicians agree to use western medicine to treat acute cardiac failure, but ten out of ten would not agree to try TCM herbal remedies (such as Gan-Cao-Gan-Jiang-Tang ) to rescue 
patients (Zhao and Fu 2008). It was described that clinicians did not like to try the TCM because they were not sure if it would work and asked "what would you do if it failed and patient died?" Then the author argued that it was as if patients did not die under the treatment of western medicine; if that was the case, "why the bigger the hospital the bigger the dead house size?" (Zhao and Fu 2008). It would be such an obvious point that the bigger the hospital, the higher proportion of severe patients, the higher general mortality for the hospital. This article demonstrated the status of evidence based practice in TCEM in China.

There are leaders in TCEM research in china who advocate the fact that without strong scientific research, TCEM would not develop further in the future in China (Chen 2001). A leading member from the Chinese Association of Combined TCM with Western Medicine reported that evidence is what TCEM needed the most at the time, such as research about time-effectiveness and dose-response of the herbal remedies (Chen 2001).

To better coordinate the research outcome, the Emergency Medicine Branch of China Traditional Chinese Medicine Association published their research priorities which were stroke, infectious fever, shock, lung diseases, cardiac attack, and virus diseases (Chao, Jiang et al. 2010). The significant research outcome in TCEM including the establishment of the TCEM peer reviewed journals such as Chinese Journal of TCM and Shanghai Journal of TCM. The first TCEM book in history titled "Traditional Chinese Emergency Medicine", over one million Chinese words, initiated and monitored by China TCM Management Bureau, was published in 1995 after three years of hard work from the TCM practitioners and researchers (Wang and Wang 1995).

The relatively active research was conducted in acupuncture due to its wide accepted effect in treating acute and chronic pain, including osteoarthritis, back pain, dysmenorrhea, and migraints (Dillard and Knapp 2005). A lot of research activities have been undertaken trying to understand the mechanism of how acupuncture works in treating pain, and they have found the improvement in immune system, neurochemical and hormone functions, and pain pathways (Dillard and Knapp 2005). However, the exact mechanism of how acupuncture works specifically remains unclear.

The challenge in further developing TCEM research included no vision and long term strategies, weakness in research to provide strong evidence, and the capacity in teaching and training to produce quality TCEM practitioners (Chao, Jiang et al. 2010).

\section{Overseas TCM}

Alternative and complementary medicine has been used among populations in a range of countries and will always be there in the future. The National Centre for Complementary and Alternative Medicine (NCCAM) outlined six categories, which are mind-body interventions, diet lifestyle modification, herbal remedies, manual healing, bioelectromgnetics, and pharmacologic-biologic treatments (Dillard and Knapp 2005). There is no doubt that TCM is one of the options when people turn to alternative and complementary medicine. For example, acupuncture gained attention in USA in the 1970s when China and USA started the diplomatic relationship (Dillard and Knapp 2005). There were approximately 10,000 certified acupuncturists practice in USA in 2005 and increasing number of those acupuncturists are physicians who have incorporated acupuncture into their daily practice (Dillard and Knapp 2005). 
TCM was commonly used among the Chinese immigrants living in other countries such as USA, Canada, UK and Australia. A survey using a convenience sample of first- and secondgeneration adult Chinese immigrants (Pearl, Leo et al. 1995) attending at an Emergency Department in a hospital in New York, found that $43 \%$ had used traditional Chinese therapy within one week of the ED visit. Therefore, emergency physicians treating ChineseAmericans should be aware of the medical and social implications of alternative medical therapies.

\section{Opportunities and challenges for the future development of emergency medicine in China among this global medicine community}

In this globalization environment, international organizations and health professionals have started to be involved in the development of EM in China. For example, a description of the US emergency physicians' experience as consultants at a new ED and in establishing an EM residency program in Hangzhou, China was reported about 13 years ago (Clem, Thomas et al. 1998). A total of seven months were spent in the observation, identification and development of a basic framework of emergency care at a new hospital in Hangzhou (Clem, Thomas et al. 1998). About six years ago, a collaborative partnership between the Johns Hopkins Hospital, Chaoyang Red Cross Hospital in Beijing and Chinese Ministry of Health was established to initiate EM administrative training in Beijing, China (Hsu, Dey et al. 2005). The Emergency Medical Education and Training Center (EMETC) at Chaoyang Red Cross Hospital was opened as a training facility to foster EM administrative curriculum development and training nationwide. A six-step approach with problem identification, needs assessment, goals and objectives, educational strategies, implementation and evaluation was used to form a locally adapted curriculum (Hsu, Dey et al. 2005). With a train-the-trainers model, the EMETC sponsored several EM administration courses, the first of their kind in China. Since its inception, the EMETC has trained 95 persons from throughout China in EM administration. An EM administration curriculum has been developed and refined. The author believes that an international partnership between academic hospitals, supported by the local Ministry of Health, to develop a national training facility using this six-step approach might be an attractive strategy for dissemination of EM administration principles (Hsu, Dey et al. 2005).

Emergency physicians from Hong Kong also believe that they could contribute to the fast development of EM as a specialty area in mainland China (Fu, Chan et al. 1998). Queensland University of Technology has signed a Memorandum of Understanding with China Medical University to work together to develop award courses and training programs in EM in China (Figure 6).

Considering the development of EM in Australia, health professionals working related to hospital EDs are well placed to contribute their knowledge and skills to assist in the development of ED in hospitals in China. As early as ten years ago, Dr Chris Curry (Curry 2001) described his vision that 'there is much new territory to be explored: in refugee and developing world medicine; in travel medicine and public health; in providing services to remote locations on the ground, water or ice; and at a distance by telemedicine. Emergency physicians have been making contributions in trouble spots like Bosnia, Timor, Myanmar, the Kenya/Sudan border, Afghanistan. We are joining with developing neighbours like New Guinea and the Pacific Islands. We are going to the outback, we are going to sea, we 


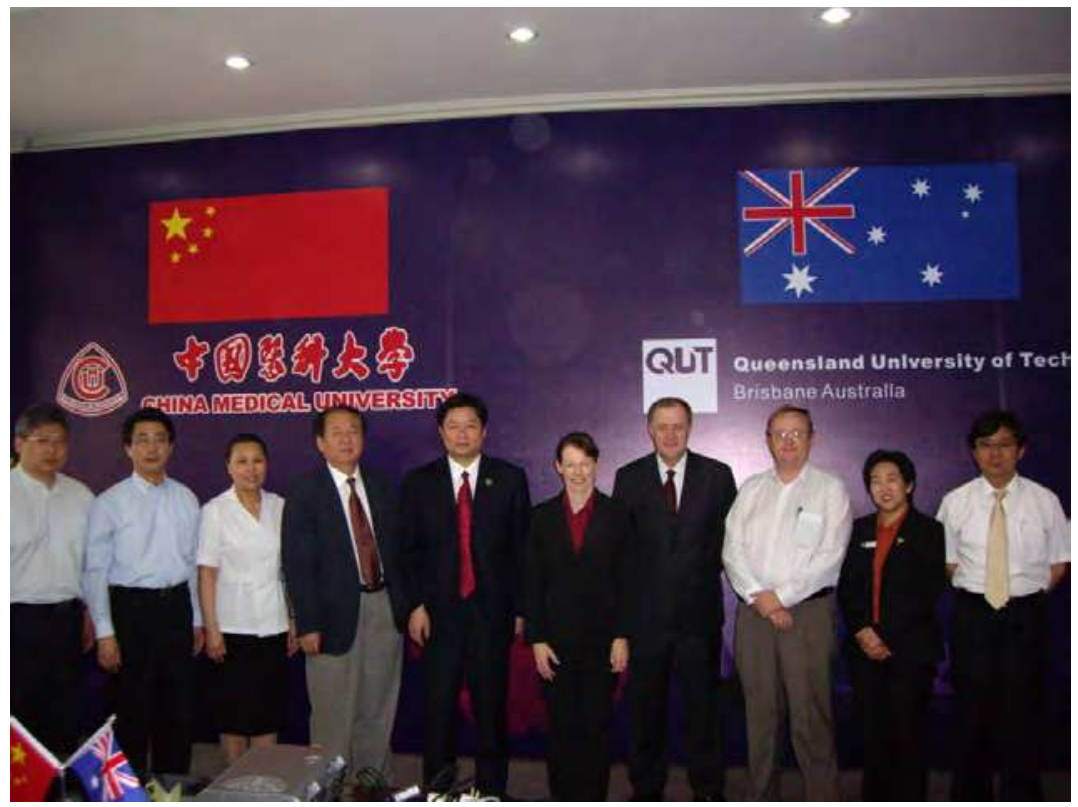

Fig. 6. QUT delegation visited China Medical University in 2006 (the second right is the author).

are developing EM links with China, South-East Asia, and the Middle East" (Curry 2001). The Australian emergency physicians are proud of their achievement, for example, Dr Cox's wonderful work in Tanzania, East Africa (Cox 2007), Australia's contribution to the EM work in post-tsunami Thailand (Liew and kennedy 2007) and Dr Frieda Law from Australia being the invited guest editor for the Chinese Journal of Emergency Medicine (Jiang 2006). Australasian emergency physicians have connections with more than 30 developing countries in the Pacific, Asia and Africa by 2006 (Curry 2007). However, we would definitely achieve more if we could link more closely to our neighbour developing countries, such as China.

The 11th National Emergency Medicine Conference was held in Dalian in May 2006 and the President Professor Jiang Guanyu expressed his excitement of holding the 17th World Congress in Disaster and Emergency Medicine in China in 2011. He expected a rapid development in EM in the coming years to prepare for the world congress, especially now China has its EM professional association, EM is a major in medicine and doctors' career path is clearer than ever (He 2006).

It is believed that TCEM will continue to provide acute care to the population in China. An health official from China TCM Management Bureau suggested to build the TCEM in the $21^{\text {st }}$ century, health professionals need to (Yang 2001) combine the tradition with innovation; the characteristics with its strength; align the ED development with the hospital development; clinical practice with clinical quality governess; research in herbal remedies; education and training in TCEM; and to combine TCEM with information technology and related regulations (Yang 2001). Modernisation was not regarded as the appropriate choice 
for TCEM's future and it actually could kill TCM as a whole (Su, Ding et al. 2002); TCM should stand on its own feet while learning the high technology from the western medicine (Su, Ding et al. 2002).

There is no doubt that China EM will develop dramatically in a short period of time, in this global environment with contributions from developed countries. Developed countries such as Australia, being China's neighbour and friend, would be well placed to play a significant role in this Emergency Medicine development.

\section{Competing interests}

I declare that there are no competing interests, such as financial support or relationships that might pose conflicts of interests during writing this book chapter.

\section{Acknowledgment}

I sincerely acknowledge the financial support from the School of Public Health at Queensland University of Technology and the generous support from Dr Jingzhou Zhao and Ms Shuang Zhong who helped me in building the Endnote library including the references in Chinese language.

\section{References}

Ali, R. (2001). "Emergency medicine in China: redefining a specialty." J Emerg Med 21(2): 197207.

Bai, X., Z. Li, et al. (2004). "The theory and practice of multi-trauma treatment in emergency department in hospitals." Chin. J. Emerg. Med 13: 863-864.

Bradt, D. A. and C. M. Drummond (2006). "Avian influenza pandemic threat and health systems response." Emerg Med Australas 18(5-6): 430-443.

Chang, L. J., Y. B. Pang, et al. (2010). "The difficulties and countermeasures of the clinical teaching to emergency nurses from TCM hospitals." China Prac Med 5(35): 261-262.

Chao, E. X., L. D. Jiang, et al. (2010). "Research report on development of the subject of emergency medicine in TCM." Chinese Medicine Modern Distance Education Of China 8(17): 164-166.

Chen, L. F., Y. Y. Wang, et al. (2011). "Discuss the training methods for core capability of nurses from emergency departments of TCM hospitals." Journal of Emergency in Traditional Chinese Medicine 20(2): 338-339.

Chen, Q. Z. (2010). "The application of the "four diagnostic" method from TCM in the triage of emergency department at a TCM Hospita." Modern Journal o $f$ Integrated Traditional Chinese and Western Medicine 19(25): 3223-3224.

Chen, S. K. (2001). "Promote the continuous development of traditional Chinese emergency medicine and related subjects." Journal of Emergency in Traditional Chinese Medicine 10(1): 2-4.

Cheng, H. and Y. H. Lu (2011). "The application of emotional nursing in 96 emergency hysteria patients." Modern Journal of Integrated Traditional Chinese and Western Medicine 20(7): 889.

China-TCM-Management-Bureau (1997). Herbal Medicine list for Emergency Departments in TCM Hospitals in China in 1997. Chin J Inf Tradit Chin Med 4: 5-6. 
China MoH. (2006). "2005 Report of China Health." from http://www.moh.gov.cn/news/search_index.aspx.

China NBoSoP. (2006). "2005 Report." from http://www.stats.gov.cn/was40/gjtjj_detail.jsp? searchword=\%BB $\% \mathrm{~A} 4 \% \mathrm{CA} \% \mathrm{BF} \&$ presearchword $=\% \mathrm{D} 2 \% \mathrm{BD}$ $\%$ C9\%FA\&channeled $=6697 \&$ record $=26$.

Clem, K. J., T. L. Thomas, et al. (1998). "United States physician assistance in development of emergency medicine in Hangzhou, China." Ann Emerg Med 32(1): 86-92.

Cox, M. (2007). "Emergency medicine development in Tanzania, East Africa." Emerg. Med. Australas 19(s1): A19.

Curry, C. (2001). "Journey with emergency medicine." Emerg. Med. Australas 13(1): 1-4.

Curry, C. (2007). "Emergency medicine in the Developing World." Emerg. Med. Australas 19(s1): A18.

David, S. (2007). "The challenges of developing emergency medicine in India." Emerg. Med. Australas 19(s1): A18.

Deng, F. Y. and A. F. Zheng (1997). "95 cases of emergency treatments of TCM for embedded hemorrhoids." Fujian Journal of Oct ober 28(6): 21.

Dillard, J. N. and S. Knapp (2005). "Complementary and alternative pain therapy in the emergency department." Emerg Med Clin North Am 23(2): 529-549.

Ding, S. X. (2005). "Suggestions for the work of emergency in TCM hospitals " Management of Chinese Medicine 4(2): 35-36.

Fan, X., C. Li, et al. (2000). "The current situation and development strategy of emergency medicine in China." J. Emerg. Med 9: 364-366.

Fu, t., K. Chan, et al. (1998). "Emergency medicine in Hong." Ann. Emerg. Med 32(1): 83-85.

Gao, Z. L. (2009). "Three ruls for the application of the angry machine method in TCM emergency." Chinese Journal of ethnomedicine and ethnopharmacy 7: 53.

He, M. F., Y. J. Zhang, et al. (2004). "Chinese and western in hand, into the modern emergency system- -the discussion of the emergency mode in TCM hospitals in Foshan city, Guangdong province." Journal of Emergency in Traditional Chinese Medicine 13(1): 43-44.

$\mathrm{He}$, X. (2006). "Report of the national 11th conference on emergency medicine." Chin. J. Emerg. Med 15: 666-668.

Hesketh, T. and W. X. Zhu (1997). "Health in China. Traditional Chinese medicine: one country, two systems." BMJ 315(7100): 115-117.

Hou, X.-Y. and K. Chu (2010). "Emergency Department in Hospitals: Window of the world A preliminary comparison between Australia and China." World Journal of Emergency Medicine 1(3): 180-184.

Hou, X. Y. and C. Z. Lu (2005). "The current workforce status of prehospital care in China." Journal of Emergency Primary Health Care 3: Article 990127.

Hsu, E. B., C. C. Dey, et al. (2005). "Development of emergency medicine administration in the People's Republic of China." J Emerg Med 28(2): 231-236.

Jelinek, G. A., P. A. Cameron, et al. (2002). "Emergency medicine." Med J Aust 176(1): 11.

Jiang, G. (2004). "The theory and practice of emergency medicine." Chin. J. Emerg. Med 13(1): 5-6.

Jiang, G. (2006). "20 years of emergency medicine in China: past and future." Chin. J. Emerg. Med 15(1): 5. 
Jiang, J. Z., H. Y. Chen, et al. (2007). "Discuss the construction of emergency departments in TCM hospitals." Journal of Emergency in Traditional Chinese Medicine 16(6): 718.

Jiang, L. C., S. Y. Tang, et al. (1992). "Application of accupressure in emergency departments at hospitals" 4(4): 239.

Jiang, S. M. (2009). "Discussion of TCM emergency " Journal of Emergency in Traditional Chinese Medicine 18(12): 1933-1934.

Jin, D. S. (1982). "The preliminary study of carrying out emergency in TCM." Hubei Journal of Traditional Chinese Medicine(5): 38-39.

Lammers, W., W. Folmer, et al. (2011). "Demographic analysis of emergency department patients at the ruijin hospital, shanghai." Emergency Medicine International 2011: 748274 .

Lan, L. H. and W. Lin (2009). "Characteristics of TCM dialectical triage and protection for emergency patients with vertigo." Journal of Liaoning University of Traditional Chinese Medicine 11: 200-201.

Li, X. L., N. L. Han, et al. (2009). "Discuss the application of PBL teaching method in the teaching of clinical emergency in TCM." Journal of Emergency in Traditional Chinese Medicine 18(10): 1663-1665.

Li, Y. (2011). "Study and practice of standardized training and assessment of clinical skills for emergency medecine of TCM " Guide of China Medicine 9(22): 176-177.

Li, Y., Y. F. Cheng, et al. (2006). "The analysis of the application of emergency necessary proprietary from TCM." Journal of Emergency in Traditional Chinese Medicine 15(4): 385-386.

Li, Y., C. Z. Yang, et al. (2010). "Clinical teaching of TCM emergency medicine applying Problem Based Learning." Education of Chinese Medicine 29(5): 69-71.

Liew, D. and M. kennedy (2007). "Emergency medicine in post-tsunami Thailand: Australia's contribution." Emerg. Med. Australas 19(s1): A8.

Lin, Y. Z. and B. Liu (2003). "Discussion on triage in emergency departments of TCM hospitals " Jilin Journal of Traditional Chinese Medicine 23(2): 41.

Little, M. and L. Murray (2004). "Consensus statement: risk of nosocomial organophosphate poisoning in emergency departments." Emerg Med Australas 16(5-6): 456-458.

Liu, L. (2010). "Strengthening the thinking for the risk management of emergency in primary TCM hospitals "Chinese Community Doctors 12(6): 161-163.

Liu, M., D. Xie, et al. (2002). "Discussion and practice of models in emergency department in TCM hospitals " Journal of Emergency in Traditional Chinese Medicine 11(5): 401-402.

Liu, Q. Q. (2008). "Get to know the science of emergentology medicine of TCM." GLOBAL TCM(1): 11-13.

Liu, Q. Q., G. L. Wang, et al. (2010). "Talk about the core of clinical teaching of TCM from emergency medicine of TCM " Chinese Medicine Modern Distance Education Of China 8(18): 196.

Liu, X. R. and C. Y. Lin (2009). "The application of "sentiment nursing" from TCM in nursing of elderly patients who are in the use of PTCA emergency care." Journal of Clinical Medicine in Practice 5(5): 32-33.

Liu, Z. H. and Y. L. Cui (2004). "Misdiagnosis and prevention of emergency medicine of TCM." Chinese Journal of Misdiagnostics 4(8): 1161-1162.

Lu, Y. and L. Qin (2005). "A Situation analysis of medical resources in emergency department in Guangxi province in China." Chin. J. Emerg. Med 14: 1054. 
Luo, K. (2010). "The application of emergency medicine and new dosage forms of TCM." Chinese Medicine Modern Distance Education Of China 8(18): 202-204.

Luo, X. (2003). "Discussions on the external environment of 'emergency department channel." Chin. J. Emerg. Med 12: 802.

Ni, L. Y. (1998). "Discuss the application of acupuncture in TCM emergency " JOURNAL OF CLINICAL ACUPUNCTURE AND MOXIBUSTION 14(1): 40-41.

Pearl, W. S., P. Leo, et al. (1995). "Use of Chinese therapies among Chinese patients seeking emergency department care." Ann Emerg Med 26(6): 735-738.

Shen, S. G. and X. J. Han (2009). "TCM emergency dialectical treatments for chest pain." Journal of Emergency in Traditional Chinese Medicine 18(10): 1628-1629.

Song, G. and X. Fan (2003). "The current situation and a few issues in emergency department in paediatrics." Chin. J. Emerg. Med 12: 293-294.

Song, W. (1999). "The theory and practice of emergency medicine development." J. Emerg. Med 8(6): 1.

Su, W. G., B. H. Ding, et al. (2002). "Applying modern emergency medicine system to promote traditional Chinese medicine." Journal of Emergency in Traditional Chinese Medicine 11(2): 77-79.

Sun, J. G. and Z. Q. Xi (2006 ). "In which direction is the way of emergency in TCM in the 21 st century?" Chinese Journal of Integrated Traditional and Western Medicine in Intensive and Critical Care 13(1): 63-64.

Sun, Z., L. Tang, et al. (2006). "The development of modern trauma rescue and treatment." Chin. J. Emerg. Med 15: 659-661.

Tan, R. Y. and G. Y. Mei (1995). "Construction and management of emergency department in TCM hospitals." Chinese Journal of Hospital Administration 11(4): 215-216.

Tao, X. Y. (2003). "The application of the "four diagnostic" from TCM in the triage of emergency abdominal pain." Journal of Emergency in Traditional Chinese Medicine 12(2): 188.

Wan, Z. and Q. He (2006). "The training of the residence doctors in emergency department in Huaxi hospital at Sichuan University " Chin. J. Emerg. Med 15: 183-185.

Wang, L. J. (2011). "The experience of "emotional nursing" for PCI patients at Emergency Department in a TCM Hospital " The Qiqihar Medical School Journal(14).

Wang, P. (2000). "Discussions on development of emergency department - to face 21st century and the newchallenges." Chin. J. Emerg. Med 9: 353-354.

Wang, R. P. and X. M. Chen (2009). "Building and the study of standardized evaluation of the green channel using emergency medicine of TCM for acute myocardial infarction." Jiangsu Journal of Traditional Chinese Medicine 41(10): 35-36.

Wang, Y. (2001). "Emergency department in city hospitals should develop a 5-year plan." Chin. J. Emerg. Med 10(1): 9-10.

Wang, Y. (2002). "To strengthen the basic construction in emergency medicine teaching: congratulations on the first bachelor course in emergency medicine in China." Chin. J. Emerg. Med 11: 272.

Wang, Z., Y. Wang, et al. (2006). "Mistakes in rescube and treatment of pesticide of organic phosphorus." Chin. J. Crit. Care Med 26: 442-443.

Wang, Z. P. and Y. Y. Wang (1995). "The first book of "Traditional Chinese Medicine (TCM) in emergency medicine "." Chinese Journal of emergency medicine 4(3): 140-141. 
Wei, H. Q. and Y. H. Su (2010). "The application of the "four diagnostic" from TCM in the evaluation of patients of emergency departments " JOURNAL OF LIAONING UNIVERSITY OF TCM 12(10): 85-86.

Wen, L. (2005). "The establishment and development in emergency department and EICU." Chin. J. Emerg. Med 14: 699-700.

$\mathrm{Wu}, \mathrm{J}$. (2009). "Problems and countermeasures of emergency in primary TCM hospitals " Journal of Emergency in Traditional Chinese Medicine 18(1): 104-105.

$\mathrm{Wu}, \mathrm{k}$. (1997). "Development in applying herbal medicine in emergency departments at TCM hospitals." Journal of Chinese tranditional emergency medical 6(6): 243.

$\mathrm{Wu}, \mathrm{Y}$. (2011). "The thinking about teaching in emergency disciplines in TCM." MEDICAL INFORMATION(9): 4519-4520.

$\mathrm{Xi}, \mathrm{Z}$. Q. (2008). "The development and prospects of emergency medicine in TCM." Journal of Emergency in Traditional Chinese Medicine 17(4): 425-427.

$\mathrm{Xu}, \mathrm{F}$. (2000). "To develop rural emergency medicine and improve quality and team management." Chin. J. Emerg. Med 9: 281-282.

Xu, Z. Y. and J. X. Liu (1994). "TCM treatments for cancer pain." Shanghai J Tradit Chin Med (12): 6-8.

YAN, X. i., T. i. X. HAN, et al. (2008). "Discussi on of treatment determination in TCM emergency, from effects of medications will be detected after the first decoction and the disease can be cured after the second decoction." JOURNAL OF TONG JI UN I VERSITY 29(4): 136-140.

Yang, R., Z. Huo, et al. (1999). "To maintain the development of emergency medicine in the medical market competition through enhancing the awareness of the practice." $J$. Emerg. Med 8(3): 1.

Yang, R. C. (2001). "Construction of the 21st century traditional Chinese emergency medicine." Zhejiang Traditional Chinese Medicine(4): 175-176.

Zhang, C. H., W. X. Zhang, et al. (2010). "Exploration and practice of the training and thinking for undergraduate students in clinical emergency of TCM." Li Shi Zhen Medicine And Materia Medicare Sesearch 21(8): 2037-2038.

Zhang, M. (2007). "The experience by applicating TCM diagnostic methods in the triage work of emergency department " Chinese Journal of Guang Ming Tranditional Chinese Medicine 22(6): 79-80.

Zhang, Q. (2010). "The thought of TCM dialectical thought and the idea of diagnosis and treatment for emergency unconcious patients " Chinese Journal of Guang Ming Tranditional Chinese Medicine 25(9): 1582-1583.

Zhang, X. J., X. L. Wang, et al. (2007). "Brief introduction of the holistic approach in Traditional Chinese Emergency Medicine." Journal of Xinjiang Tranditional Chinese Medicine 25(4): 112-113.

Zhao, B. and Z. P. Fu (2008). "The status quo and thought of the work of emergency in TCM in Gansu." Gansu Journal of TCM 21: 54-55.

Zhong, M. L. (1983). "Preliminary discussion of dialectical emergency medicine treatment of TCM " Qinghai Medicine 61(3): 58-64.

Zhou, H. P. (1996). "Emergency medicine of TCM for 30 years." The new journal of traditional Chinese medicine 1(1): 37.

Zhou, J., S. Chen, et al. (2004). "The Investigation of chemical poisoning in emergency department in 25 comprehensive hospitals." Chin. J. Emerg. Med 13: 729-732. 
Zhou, S. B. and W. Q. Zhang (2011). "The discussion of clinical teaching in emergency department of TCM hospitals " Chinese Journal of Traditional Chinese Medicine 9(16): 46-47.

Zhou, X. P. (2007). "Discuss the application of "seven emotions" to cure and cause diseases into TCM emergency nursing." Journal of Guiyang College of Traditional Chinese Medicine 29(6): 69. 


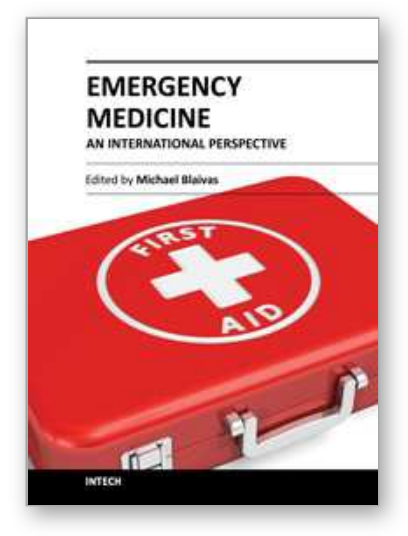

\author{
Emergency Medicine - An International Perspective \\ Edited by Dr. Michael Blaivas
}

ISBN 978-953-51-0333-2

Hard cover, 220 pages

Publisher InTech

Published online 16, March, 2012

Published in print edition March, 2012

Emergency Medicine is an expanding field that has spread beyond the shores of North America and has taken on different characteristics around the world. Although many of the struggles of emergency practitioners are similar, the field and its principles have adapted to local needs and resources. This book seeks to educate readers not only on emergency medicine theory, science and practice, but also reflects that multinational nature of emergency medicine, allowing readers to learn from experiences of others. This diverse group of authors presents a true international view of emergency medicine practice and science that will be educational for any reader.

\title{
How to reference
}

In order to correctly reference this scholarly work, feel free to copy and paste the following:

Xiang-Yu Hou (2012). Emergency Medicine in China, Emergency Medicine - An International Perspective, Dr. Michael Blaivas (Ed.), ISBN: 978-953-51-0333-2, InTech, Available from:

http://www.intechopen.com/books/emergency-medicine-an-international-perspective/emergency-medicine-inchina

\section{INTECH}

open science | open minds

\section{InTech Europe}

University Campus STeP Ri Slavka Krautzeka 83/A 51000 Rijeka, Croatia

Phone: +385 (51) 770447

Fax: +385 (51) 686166 www.intechopen.com

\section{InTech China}

Unit 405, Office Block, Hotel Equatorial Shanghai No.65, Yan An Road (West), Shanghai, 200040, China 中国上海市延安西路65号上海国际贵都大饭店办公楼 405 单元 Phone: +86-21-62489820

Fax: +86-21-62489821 
(C) 2012 The Author(s). Licensee IntechOpen. This is an open access article distributed under the terms of the Creative Commons Attribution 3.0 License, which permits unrestricted use, distribution, and reproduction in any medium, provided the original work is properly cited. 